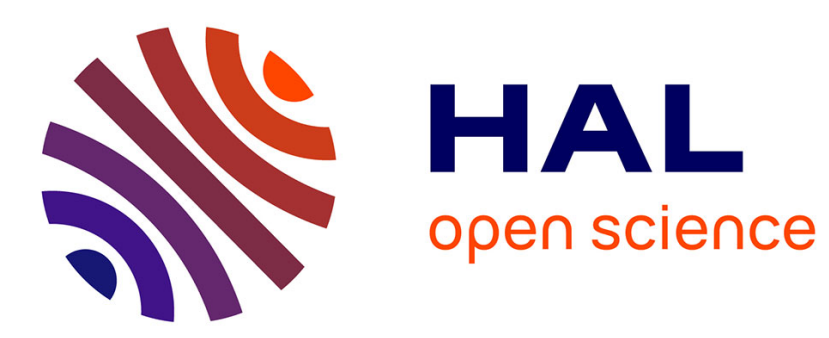

\title{
THE INFLUENCE OF COLLISIONAL DAMPING ON SURFACE PLASMON-POLARITON DISPERSION
}

\author{
A. Boardman, P. Egan
}

\section{To cite this version:}

A. Boardman, P. Egan. THE INFLUENCE OF COLLISIONAL DAMPING ON SURFACE PLASMON-POLARITON DISPERSION. Journal de Physique Colloques, 1984, 45 (C5), pp.C5-179-

C5-190. 10.1051/jphyscol:1984526 . jpa-00224144

HAL Id: jpa-00224144

https://hal.science/jpa-00224144

Submitted on 1 Jan 1984

HAL is a multi-disciplinary open access archive for the deposit and dissemination of scientific research documents, whether they are published or not. The documents may come from teaching and research institutions in France or abroad, or from public or private research centers.
L'archive ouverte pluridisciplinaire $\mathbf{H A L}$, est destinée au dépôt et à la diffusion de documents scientifiques de niveau recherche, publiés ou non, émanant des établissements d'enseignement et de recherche français ou étrangers, des laboratoires publics ou privés. 
THE INFLUENCE OF COLLISIONAL DAMPING ON SURFACE PLASMON-POLARITON DISPERSION

\author{
A.D. Boardman and P. Egan \\ Department of Pure and Applied Physics, University of Salford, \\ Salford ME 4WT, U.K. \\ Résumé - Nous présentons une analyse détaillée, analytique \\ et numérique, de l'influence conjointe de l'amortissement \\ dû aux collisions et de la dispersion spatiale sur les \\ plasmons-polaritons de surface. On trouve que les courbes \\ de dispersion présentent plusieurs branches, dont l'une est \\ le mode couramment admis comme étant à inversion de courbure \\ et qui correspond à une fréquence réelle et un nombre d'onde \\ complexe. On montre qu'une interaction importante entre l'amor- \\ tissement dû aux collisions et la dispersion spatiale peut \\ supprimer cette inversion de courbure. \\ Abstract - A detailed analytical and numerical analysis is given of \\ the joint influence of collisional damping and spatial dispersion \\ on surface plasmon-polaritons. It is shown that the dispersion has \\ several branches, one of which is the currently accepted bend-back \\ mode that arises under real frequency and complex wave number \\ assumptions. It is proved that an important interaction between \\ collisional damping and spatial dispersion occurs that can cause \\ this bend-back to be suppressed.
}

1 - INTRODUCTION

The inclusion of collisional damping into a description of surface $/ 1,2,3,4 /$ plasmon-polaritons has been discussed several times in the literature $/ 1,2 \%$. It is not a straightforward matter, especially if the model is related to attenuated total reflection (ATR) experiments /2,5/.

At the basic level, if collisions are accounted for in a model of the electron gas then the resulting surface polariton dispersion equation becomes complex. The wave number $k$ and angular frequency $w$ are then, in principle, both complex. The solution of the dispersion equation with complex $k$ and real $\omega$ or complex $\omega$ and real $k$ are not trivially distinct possibilities. Indeed, if spatial dispersion is ignored then the current position embraces the following situations. If $\omega$ is real and $k$ is complex, and equal to $k_{r}+i k_{j}$, a plot of wagainst $k_{r}$ follows the usual collisionless surface polariton dispersion curve upto the region $\omega \omega_{p} / \sqrt{ } 2$, where $\omega_{p}$ is the plasma frequency. It then bends back towards the light line. This has been confirmed experimentaliy for silver $/ 6 /$. If $\omega$ is complex, and equal 
to $\omega_{r}+i \omega_{i}$ and $k$ is real then a plot of $\omega_{r}$ against $k$ does not show this bend-back and follows the collisionless surface polariton curve. The first possibility corresponds to an ATR measurement in which the excitation frequency is fixed and the angle of incidence is scanned while the second corresponds to a fixed angle of incidence with an excitation frequency scan.

It is the bend-back that is of the greatest theoretical interest and it is important to discover what happens when spatial dispersion is included. Part of the answer suggests itself immediately. At high enough wave numbers in the electrostatic region the $\left(\omega, k_{r}\right)$ dispersion diagram must have a spatial dispersion branch even if at lower wave numbers it is suspected that bend-back can still occur. The detailed answer is a complicated one. It is shown in this paper that bend-back can still occur but, even though the wave numbers in the region over which it occurs are small, it can be strongly influenced by spatial dispersion. Indeed if this influence is strong enough it can isolate and suppress the phenomenon.

The presentation here concentrates on three $\left(\omega / \omega_{p}\right)^{2}$ regions namely $0<\Omega^{2}<0.4, \Omega=\omega / \omega_{p}, 0 . A<\Omega^{2}<0.6$ and $0.6<\Omega^{2}<0.9$. In the first and last region analytical results are obtained. These then provide an interpretation for the centre region that has to be investigated computationally. The outside regions, therefore, continue in terms of labelling into the centre region. The problem is conveniently analysed in terms of $N^{2}=c^{2} k^{2} / \omega^{2}$, where $c$ is the velocity of light in vacuo. The conclusions drawn are then finaliy translated into $\omega, k_{r}$ plots in which the features discussed above are clearly displayed.

\section{II - THE DISPERSION EQUATION}

If both spatial dispersion and damping are accounted for in a hydrodynamic description of the electron gas then the bulk transverse wave number $k_{T}$ and the bulk longitudinal wave number $k_{L}$ are, respectively, given by $/ 1,4 /$

$$
k_{T}^{2}=\omega^{2} / c^{2}\left|1-\omega_{p}^{2} / \omega(\omega+i v)\right|
$$

and

$$
1-\omega_{p}^{2} /\left(\omega^{2}+i v \omega-\beta^{2} k_{L}^{2}\right)=0
$$

where the time dependence is assumed to be $\mathrm{e}^{-i \omega t}, \omega$ is the angular frequency, $v$ is a constant electron collision frequency and $B^{2}=\frac{3}{5} v_{F}^{2}$ where $V_{F}$ is the Fermi velocity. The surface mode dispersion equation for a vacuum-bounded semi-infinite plasma, derived earlier in a different way by Clemmow and Elgin /l/ for a gaseous electron plasma, is $\left\{\omega^{2}-\omega_{p}^{2} /(1+i \nu / \omega)\right\}\left(c^{2} k^{2}-\omega^{2}\right)^{\frac{1}{2}}+\omega^{2}\left(c^{2} k^{2}-\omega^{2}+\omega_{p}{ }^{2} /(1+i \nu / \omega)\right)^{\frac{1}{2}}$ 


$$
-\frac{c^{2} k^{2} \beta \omega_{p}^{2}}{(1+i \nu / \omega)\left(B^{2} k^{2}-\omega^{2}\left(1+i \nu / \omega_{\rho}+\omega_{p}{ }^{2}\right)\right.}=0
$$

This dispersion equation involves radicals and is beyond ordinary analytical methods of determining the roots. As is shown below the 'squaring up' of the equation, very fortunately, leads to a cubic equation. The roots of this equation can, of course, be written down but may or may not be physically admissible. It is necessary then to develop a root 'acceptance' criterion. Part of the algorithm can be physical; the main approach however, is to check whether the root satisfies the original dispersion equation. If $N=c^{2} k^{2} / \omega^{2}=\left(\frac{c k}{\omega_{p}}\right)^{2}\left(\frac{\omega_{p}}{\omega}\right)^{2}=\frac{k^{2}}{\Omega^{2}}$, where $k=c k / \omega_{p}$ and $\Omega=\omega / \omega_{p}$, then equation (2.3) reduces to the cubic

$$
a N^{3}-b^{2} N^{2}-2 b d N-d^{2}=0
$$

where

$$
a=4 \xi^{2} \Omega^{3}(\Omega+i n), b=\zeta^{2} \Omega^{2}+2 \Omega^{2}-1+2 i \Omega n, d=l-\Omega^{2}-i \Omega n
$$

and $n=v / \omega_{p}, \xi=B / c$. If $N=x+\frac{b^{2}}{3 a}$ is introduced to equation (2.4) it reduces even further to

$$
x^{3}+q x+r=0
$$

where

$$
q=-\frac{b}{3 a}\left(b^{3}+b a d\right), r=-\left(2 b^{6}+18 a b^{3} d+27 a^{2} d^{2}\right) / 27 a^{3}
$$

Equation (2.6) has solutions

$$
x=\left[-\frac{r}{2}+r \frac{r^{2}}{4}+\frac{q^{2}}{27}\right]^{1 / 3}+\left[-\frac{r}{2}-r \frac{r^{2}}{4}+\frac{q^{2}}{27}\right]^{1 / 3}=y+z
$$

The cube roots must now be chosen to make $y z=-\frac{q}{3}$ i.e. $y z$ is rational Hence the three roots required are $y+z, \lambda y+\lambda^{2} z$ and $\lambda^{2} y+\lambda z$ where $\lambda$ and $\lambda^{2}$ are the two complex roots of 1 i.e. $-\frac{1}{2}(1-i \sqrt{3})$ and $-\frac{1}{2}(1+i \sqrt{3})$. The general solution is therefore

$$
\begin{aligned}
3 y^{\prime} 2 a N= & 2^{\frac{1}{3}} b^{2}+\left\{2 b^{3}\left(b^{3}+9 a d\right)+27 a^{2} d^{2}+\sqrt{27 a^{3} d^{3}\left(4 b^{3}+27 a d\right)}\right\}^{1 / 3} \\
& +\left\{2 b^{3}\left(b^{3}+9 a d\right)+27 a^{2} d^{2}-\sqrt{27 a^{3} d^{3}\left(4 b^{3}+27 a d\right)}\right\}^{1 / 3}
\end{aligned}
$$

This equation does not involve any approximation and can therefore be used to study the damped and undamped surface modes.

III - ZERO DAMPING

Although this case has been discussed in a general form before, it is true to say that the discussion was very limited and that a full and unambiguous development has not been previously given. Here the meaning of various branches of the dispersion diagram is derived and a clear indication of which of the curves can be labelled as surface waves is given. The 
representation adopted here is a useful and revealing one that allows the role of collisions and spatial dispersion to be understood quite clearly. Let us first consider the solutions in the region I i.e. $0<\Omega^{2}<0.4$. This region is chosen to make absolutely certain that the analytical work is valid but in practice the upper limit could be raised. In this region we skirt to the left of the problematic point $\Omega^{2} \approx 0.5$ that would be singular if $\xi \rightarrow 0$ and $n \rightarrow 0$. In this region $\left|b^{3}\right|>8 \times 10^{-3}$, $a d \leqslant 4 \times 10^{-7}$ and $(a b d)^{3 / 2} \leqslant 10^{-12}$. Hence if

$$
\begin{aligned}
& p=2 b^{6}+9 a d\left(2 b^{3}+3 a d\right) \\
& q^{\prime}=\left|27 a^{3} d^{3}\left(4 b^{3}+27 a d\right)\right|
\end{aligned}
$$

then $p>>q^{\prime}$ and we have for the roots inside \{\}

$$
\begin{aligned}
& \left(p+i q^{-\frac{1}{2}}\right)^{\frac{1}{3}}+\left(p-i q^{-\frac{1}{2}}\right)^{\frac{1}{3}}=\frac{7}{3 p^{2}}\left[\left(3 p+i q^{-\frac{1}{2}}\right)+\left(3 p-i q^{-\frac{3}{2}}\right)\right] \\
& 1 /\left(6 p^{2 / 3}\right)\left[\left(3 p+i q^{-\frac{2}{2}}\right)(-1+i \sqrt{3})+\left(3 p-i q^{-\frac{1}{2}}\right)(-1-i \sqrt{3})\right] \\
& 1 /\left(6 p^{2 / 3}\right)\left[\left(3 p+i q^{-\frac{1}{2}}\right)(-1-i \sqrt{3})+\left(3 p-i q^{-\frac{1}{2}}\right)(-1+i \sqrt{3})\right]
\end{aligned}
$$

so that the three roots $\mathrm{N}_{1}, \mathrm{~N}_{2}$ and $\mathrm{N}_{3}$ are given by

$$
3(2)^{1 / 3} a N_{1}=2^{2 / 3} b^{2}+p^{1 / 3}, \quad 3(2)^{1 / 3} a N_{2 / 3}=2^{1 / 3} b^{2}-p^{1 / 3}+1-1-\sqrt{3} q^{-1 / 2} /\left(3 p^{2 / 3}\right)
$$

where $2 / 3$ refers to $t /-$. Hence

$$
N_{1}=\left(2 \Omega^{2}-1\right)^{2} / 4 \xi^{2} \Omega^{4}, \quad N_{2 \beta}=\frac{\Omega^{2}-1}{2 \Omega^{2}-1}+1-\frac{2 \Omega^{2} \xi\left(1-\Omega^{2}\right)}{\left(2 \Omega^{2}-1\right)^{2}}\left|\frac{\left(1-\Omega^{2}\right)}{2 \Omega^{2}-1}\right|^{\frac{1}{2}}
$$

Here $N_{1}$ gives the large $K$ approximation and $N_{2 / 3}$ are small perturbations of $0(\xi)$ from the cold plasma case and are, in fact, the first two terms of an expansion, given in a different form, by Clemmow and Elgin $/ 1 /$.

The attenuation coefficients of a vacuum bounded semi-infinite plasma are $\alpha, \gamma$ and $\delta$ where $/ 4 /$

Vacuum: $\quad \delta^{2}=K^{2}-\Omega^{2}$

Plasma: $\quad \alpha^{2}=1+K^{2}-\Omega^{2}$

$$
\gamma^{2}=\left(\xi^{2} K^{2}+1-\Omega^{2}\right) / \xi^{2}
$$

If $\mathrm{N}_{1}$ is used then

$$
\alpha_{1}= \pm\left(2 \Omega^{2}-1\right) / 2 \Omega \xi, \delta_{1}= \pm\left(2 \Omega^{2}-1\right) / 2 \xi \Omega, \gamma_{1}= \pm 1 / 2 \Omega \xi
$$

where the signs are arbitrary and independent. For a surface wave a dispersion equation is arrived by requiring that $\alpha_{1}>0, \xi_{3}>0$ so the negative signs must be chosen in equation ( 3.11$)$. The dispersion equation in the collisionless case reduces to 


$$
\Omega^{2} \alpha-\left(1-\Omega^{2}\right) \delta=K^{2} / \gamma
$$

Hence on substitution of $\alpha_{1}$ and $\delta_{1}$ the left-hand side of (3.12) gives

$$
L_{1}=\frac{\left(1-2 \Omega^{2}\right)}{2 \Omega \xi}\left(2 \Omega^{2}-1\right)<0
$$

and the right hand side gives

$$
R_{1}=\Omega^{2}\left(2 \Omega^{2}-1\right)^{2} / 4 \xi^{2} \Omega^{4} \gamma_{1}
$$

But we only obtain $R_{1}<0$ when $\gamma_{1}<0$. This means that $N_{1}$ cannot represent a surface wave solution.

For $\mathrm{N}_{2 / 3}$ we obtain

$$
\begin{aligned}
& \delta_{2 / 3}= \pm \Omega^{2}\left(1-2 \Omega^{2}\right)^{\frac{1}{2}}\left[1+1-\xi\left(1-\Omega^{2}\right)^{3 / 2} /\left(1-2 \Omega^{2}\right)^{3 / 2}\right] \\
& \alpha_{2 / 3}= \pm 1 /\left(1-2 \Omega^{2}\right)^{\frac{1}{2}}\left[1-\Omega^{2}+/-\Omega^{4}\left(1-\Omega^{2}\right)^{\frac{1}{2}} \xi /\left(1-2 \Omega^{2}\right)^{3 / 2}\right] \\
& \gamma_{2 / 3}= \pm\left(1-\Omega^{2}\right)^{\frac{1}{2}} / \xi
\end{aligned}
$$

Now using positive signs for $\alpha_{2 / 3}$ and $\delta_{2 / 3}$ a substitution into the dispersion equation results in

$$
\Omega^{2} \alpha-\left(1-\Omega^{2}\right) \delta=+/-\left(-\Omega^{2}\left(1-\Omega^{2}\right)^{\frac{1}{2}} /\left(1-2 \Omega^{2}\right)\right)
$$

This is $<0$ for $N_{2}$ and $>0$ for $N_{3}$, so only $N_{3}$ represents a surface wave in this region. Note that $N_{3}$ lies below the cold plasma solution. We turn now to the analytically secure region III i.e. $0.6<N^{2}<0.9$ where, once again, the lower limit is approximate and can be lowered. In this region

$$
N_{1}=\left(2 \Omega^{2}-1\right) / 4 \xi^{2} \Omega^{4}, \quad N_{2 / 3}=-\left(1-\Omega^{2}\right) /\left(2 \Omega^{2}-1\right)+1-2 i \Omega^{2} \xi\left(1-\Omega^{2}\right)^{3 / 2} /\left(2 \Omega^{2}-1\right)^{5 / 2}
$$

For $N_{1}, \alpha_{1}, \delta_{1}>0$ with the positive sign in equation (3.11). This makes $L_{1}>0$ which matches $R_{1}$ for $\gamma_{1}>0$. Hence $N_{1}$ represents a surface wave solution. For region III we also obtain

$$
\begin{aligned}
& \alpha_{2 / 3}= \pm 1 /\left(2 \Omega^{2}-1\right)^{\frac{1}{2}}\left[-1+\Omega^{4}\left(1-\Omega^{2}\right)^{\frac{3}{2}} \xi /\left(2 \Omega^{2}-1\right)^{3 / 2}+i\left(1-\Omega^{2}\right)\right] \\
& \delta_{2 / 3}= \pm \Omega^{2} /\left(2 \Omega^{2}-1\right)^{\frac{1}{2}}\left[-1+\left(1-\Omega^{2}\right)^{3 / 2} \xi /\left(2 \Omega^{2}-1\right)^{3 / 2}+i\right]
\end{aligned}
$$

For $\mathrm{N}_{3}, R(\alpha)>0, Q(\delta)>0$ provided that the positive root is taken so that

$$
\alpha_{3}=1 /\left(2 \Omega^{2}-1\right)^{\frac{1}{2}}\left[\Omega^{4}\left(1-\Omega^{2}\right)^{\frac{1}{2}} \xi /\left(2 \Omega^{2}-1\right)^{\frac{3}{2}}+i\left(1-\Omega^{2}\right)\right]
$$




$$
\delta_{3}=\Omega^{2} /\left(2 \Omega^{2}-1\right)^{\frac{1}{2}}\left[\left(1-\Omega^{2}\right)^{\frac{3}{2}} \xi /\left(2 \Omega^{2}-1\right)^{\frac{3}{2}}+i\right]
$$

This choice leads to

$$
L_{1}=\Omega^{2}\left(1-\Omega^{2}\right)^{\frac{1}{2}} \xi_{3} /\left(2 \Omega^{2}-1\right)>0
$$

and

$$
R_{1}=-\left(1-\Omega^{2}\right) \xi /\left[\left(2 \Omega^{2}-1\right)\left(1-\Omega^{2}\right)^{\frac{1}{2}}\right]<0
$$

so that $\mathrm{N}_{3}$ cannot represent a surface wave. In the same way taking negative signs for the roots in question (3.19) and (3.22) to keep $Q(\alpha), Q(\delta)>0$ only changes the imaginary parts of $\alpha$ and $\delta$ which then cancel out. Hence $N_{2}$ is also not a surface wave. This means that in the collisionless spatially dispersive plasma only $\mathrm{N}_{3}$ shown in Fig.l represents a surface wave in the region I below $\Omega^{2} \approx 0.5$ while $\mathrm{N}_{1}$ represents a surface wave in region III above $\Omega^{2} \approx 0.5$. The above technique will now be used to analyse the much more difficult case when collisional damping is included.

IV - EFFECT OF COLLISIONAL DAMPING

The effects of damping may be introduced through the transformations



Fig. I - The real part of the roots of equations (2.4) for the collisionless case with spatial dispersion. 


$$
\omega_{p}^{2} \rightarrow \omega_{p}^{2} /(1+i v / \omega), \quad \xi^{2} \rightarrow \xi^{2} /(1+i v / \omega)
$$

The coefficients of the cubic equation become $a=4 \xi^{2} \Omega^{3}(\Omega+i n), b=\xi^{2} \Omega^{2}+$ $2 \Omega \Omega^{2}-1+2 i n \Omega, c=1-\Omega^{2}-i \Omega n$. The development is now restricted to first order in $\xi$ and $n$ which is a valid approximation provided that $\Omega^{2}$ does not approach too closely to $I$ in region III. In both regions I and III

$$
N_{1}=\left(2 \Omega^{2}-1\right)^{2} / 4 \xi^{2} \Omega^{4}+i n\left(4 \Omega^{4}-1\right) / 4 \xi^{2} \Omega^{5}
$$

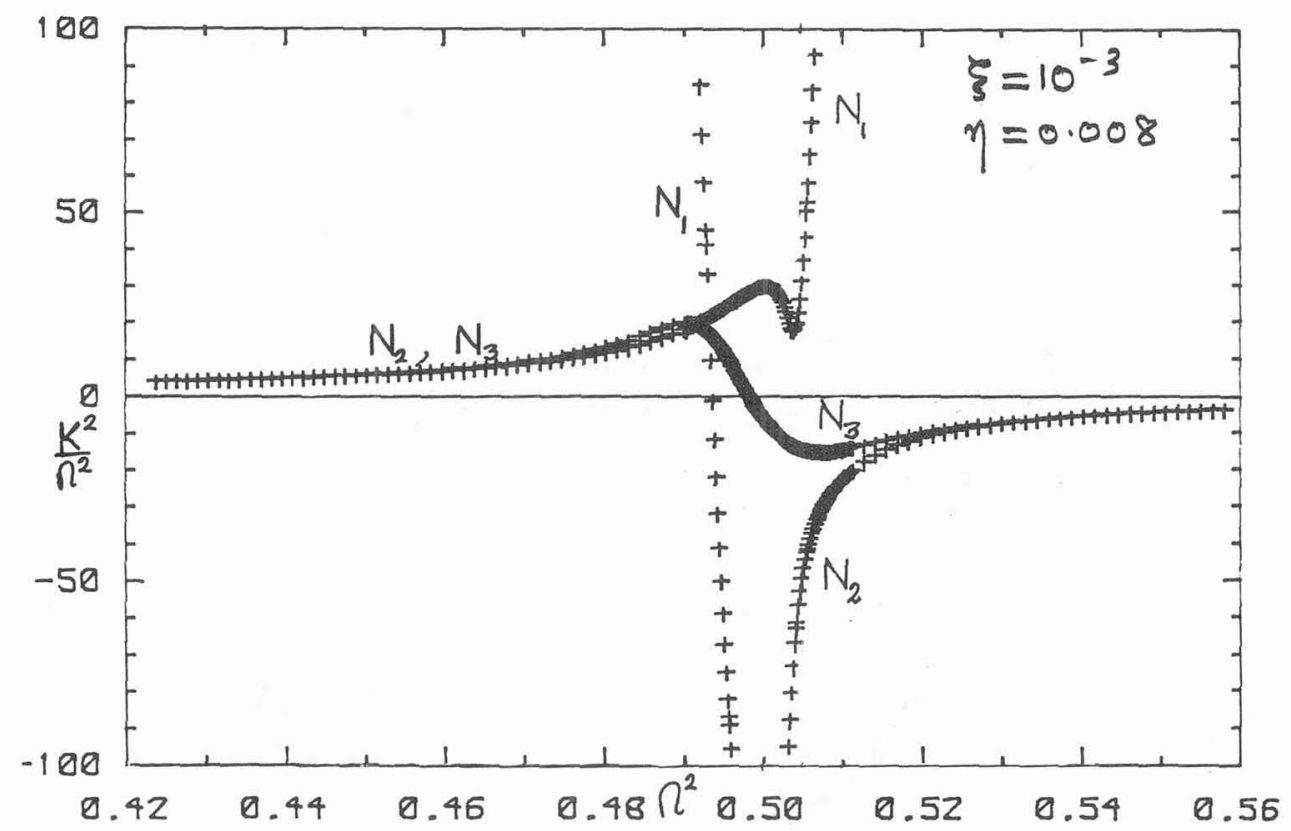

Fig. 2 - Effect of modest collisional damping on the real part of the roots of equation (2.4). Note the 'kink' in the $N_{1}$ branch.

$$
\alpha_{1}= \pm\left\{\left(2 \Omega^{2}-1\right) / 2 \Omega \xi+i n / \xi\right\}, \delta_{1}= \pm\left\{\left(2 \Omega^{2}-1\right) / 2 \Omega \xi+i n / \xi\right\}, \gamma_{1}= \pm 1 / 2 \Omega \xi
$$

Here, for convenience, we introduce the artificial transformations $\alpha \rightarrow \frac{\sqrt{2+i n}}{\Omega} \alpha$, $\delta \rightarrow \sqrt{\frac{\Omega+i n}{\Omega}} \delta, \quad \gamma+\sqrt{\frac{\Omega+i n}{\Omega}} \gamma$ that convert the real and imaginary parts of the dispersion equation, after writing $\alpha=\alpha^{\prime}+i \alpha^{\prime \prime}, \delta=\delta^{\prime}+i \delta^{\prime \prime}$, $\gamma=\gamma^{\prime}+i \gamma^{\prime \prime}$ and $K=K^{\prime}+i K^{\prime \prime}$, into

$\Omega^{2}\left(\alpha^{\prime} \Omega-\alpha^{\prime \prime} n\right)-\Omega\left[\left(1-\Omega^{2}\right) \delta^{\prime}+\Omega n \delta^{\prime \prime}\right]=\left[\gamma^{\prime}\left(K^{\prime 2} \Omega-K_{n}^{\prime \prime 2}\right)+\gamma^{\prime \prime}\left(K^{\prime \prime 2} \Omega+K^{\prime 2} n\right)\right] /\left(\gamma^{2}+\gamma^{\prime \prime 2}\right)$

$\Omega^{2}\left(\Omega \alpha^{\prime \prime}+\alpha^{\prime} \eta\right)-\Omega\left[\left(1-\Omega^{2}\right) \delta^{\prime \prime}-\Omega n \delta^{\prime}\right]=\left[\gamma^{\prime \prime}\left(K^{\prime \prime 1^{2}}+K^{\prime 2} n\right)-\gamma^{\prime \prime}\left(K^{\prime} \Omega-K^{\prime \prime} h\right)\right] /\left(\gamma^{2}+\gamma^{\prime \prime 2}\right)$

Since we are working to $O(n)$ this means that the true decay functions can 


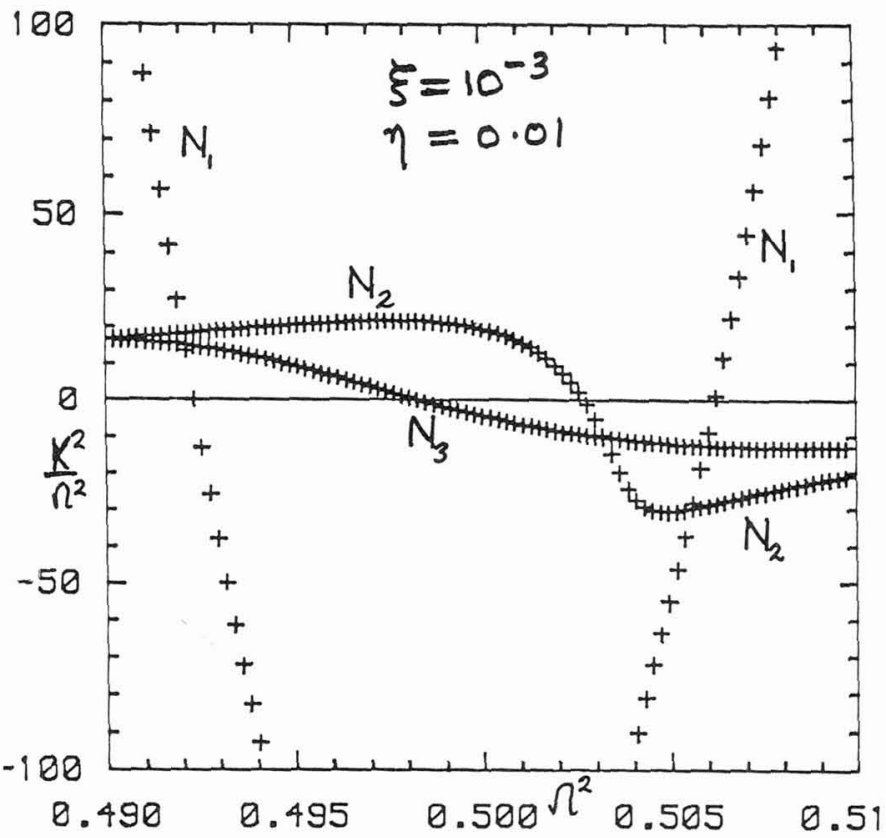

Fig. 3 - Development of the real parts of the roots of equation (2.4) as the collisional damping gets stronger.

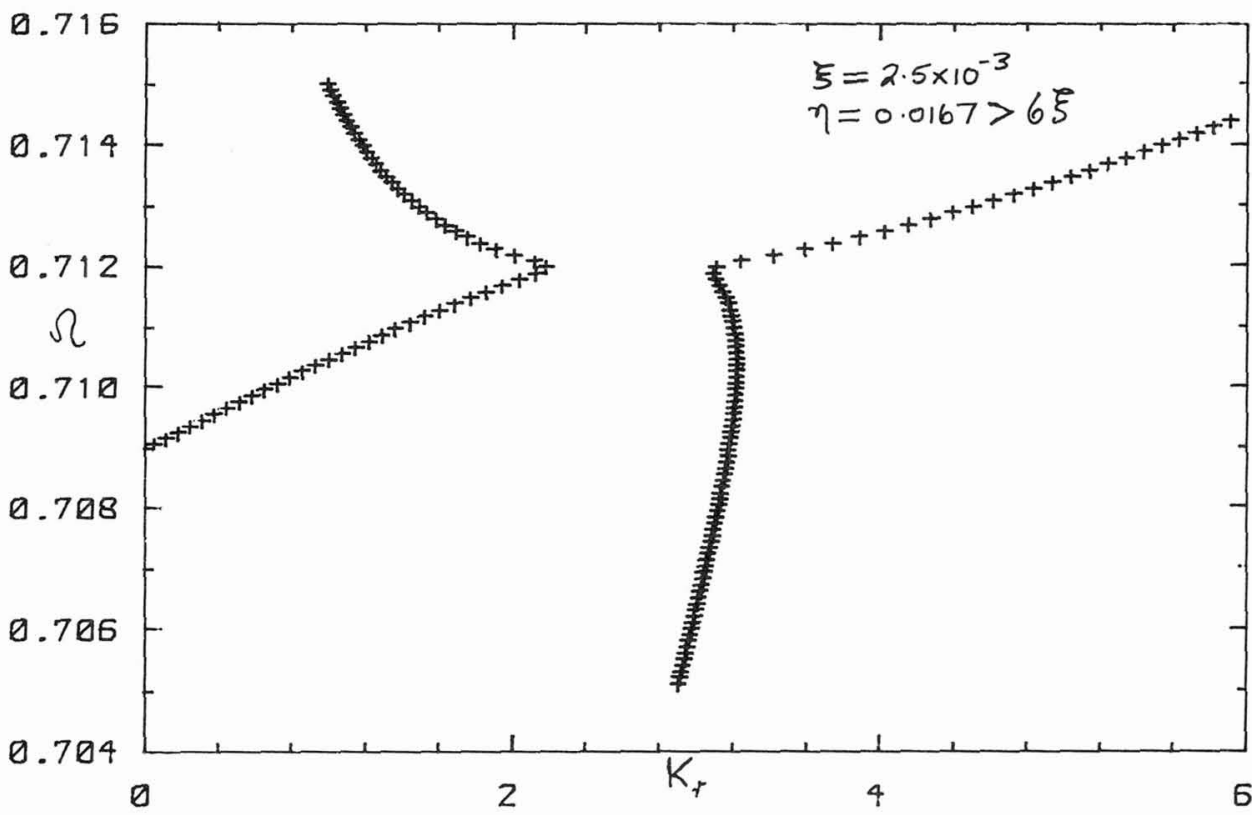

Fig. 4 - Dispersion diagram showing $\Omega$ as a function of $k_{r}$ the real part of $k$. Here $\eta$ is close to $\simeq 6 \boldsymbol{\xi}$ so there is strong interaction between the collisional and the spatial dispersion branch. 

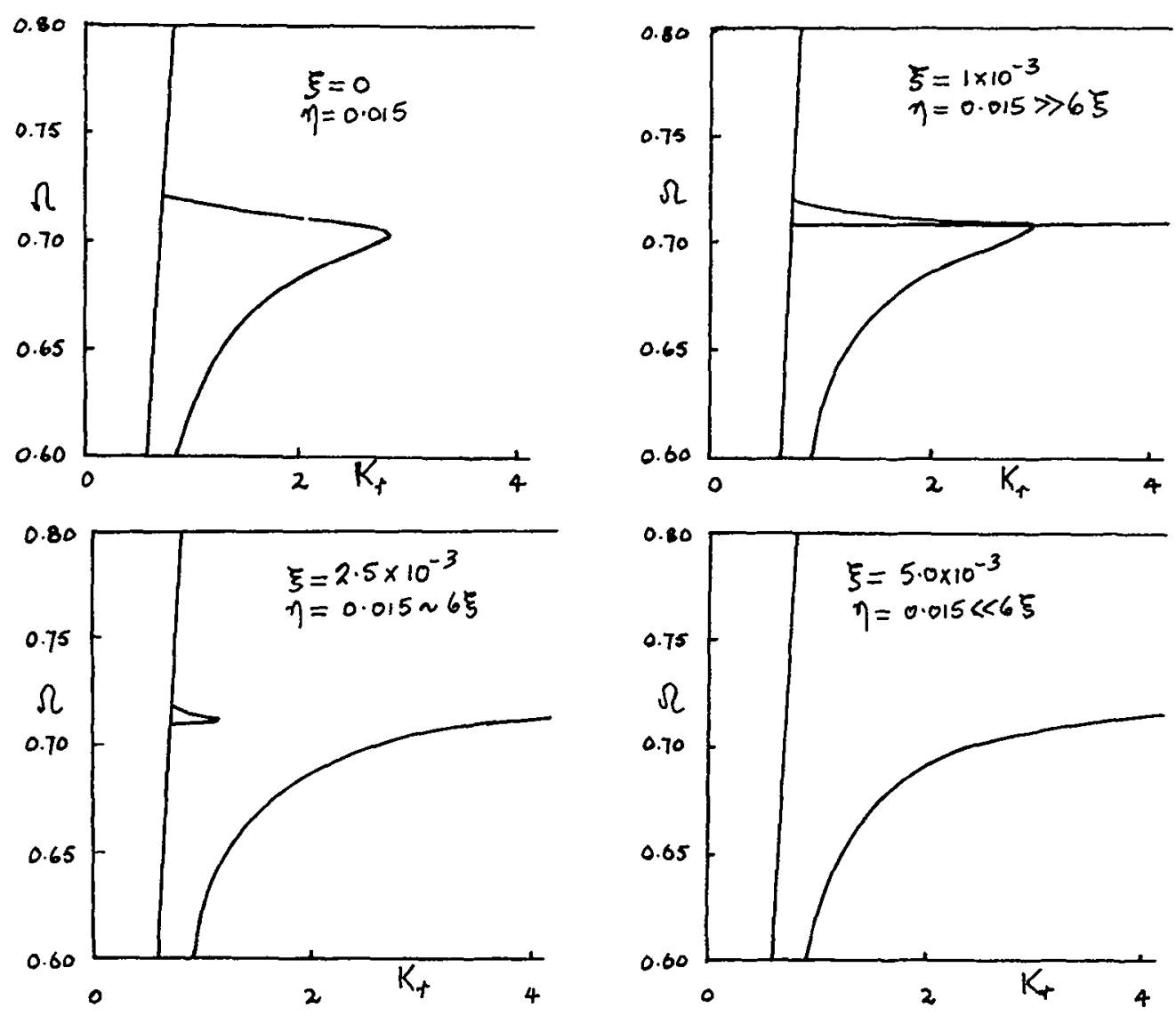

Fig. 5 - A complete progression of dispersion diagrams from $\boldsymbol{\xi}=0, \eta \neq 0$
showing the ultimate suppression of the bend-back.

be recovered after multiplying through with a factor $(1-2 i n / \Omega)$. The presence of this factor does not, in any way, a1ter the process of sorting out which branch represents a surface wave.

For the root $N_{1}$ we have from the left $\left(L_{1}\right)$ and right $\left(R_{1}\right)$ hand side of equations (4.4)

$$
\begin{aligned}
& L_{1}= \pm 1 /(2 \Omega \xi)\left[\left(2 \Omega^{2}-1\right)^{2}+4 i \Omega n\left(2 \Omega^{2}-1\right)\right] \\
& \mathrm{R}_{1}=1 /\left(4 \xi^{2} \Omega \gamma_{1}\right)\left[\left(2 \Omega^{2}-1\right)^{2}+4 i \Omega n\left(2 \Omega^{2}-1\right)\right]
\end{aligned}
$$

These equations show that for $\left(\alpha_{1}\right)>0,\left(\delta_{1}\right)>0$ the dispersion equation is satisfied only when $2 \Omega^{2}>1$. Therefore, as in the collisionless case 
$\mathrm{N}_{1}$ represents a surface wave onty in region $\mathrm{ItI}$.

In region $I \quad\left(0 \leqslant \Omega^{2} \leqslant 0.4\right)$

$$
\begin{aligned}
& N_{2 / 3}=\left(1-\Omega^{2}\right) /\left(1-2 \Omega^{2}\right)+/-2 \Omega^{2} \xi\left(1-\Omega^{2}\right)^{3 / 2} /\left(1-2 \Omega^{2}\right)^{5 / 2}+i \Omega n /\left(1-2 \Omega^{2}\right)^{2} \\
& \alpha_{2 / 3}= \pm\left\{\left(1-\Omega^{2}\right) /\left(1-2 \Omega^{2}\right)^{\frac{1}{2}}-1+\Omega^{4} \xi\left(1-\Omega^{2}\right)^{\frac{1}{2}} /\left(1-2 \Omega^{2}\right)^{2}+i \Omega^{3} n /\left(1-2 \Omega^{2}\right)^{\frac{3}{2}}\right\} \\
& \delta_{2 / 3}= \pm\left\{\Omega^{2} /\left(1-2 \Omega^{2}\right)^{\frac{3}{2}}-1+\Omega^{2} \xi\left(1-\Omega^{2}\right)^{3 / 2} /\left(1-2 \Omega^{2}\right)+i \Omega n\left(1-\Omega^{2}\right) /\left(1-2 \Omega^{2}\right)^{3 / 2}\right\} \\
& \gamma_{2 / 3}= \pm\left(1-\Omega^{2}\right)^{\frac{1}{2}} / \xi\left[1+i n\left(1-2 \Omega^{2}\right) /\left[2 \Omega\left(1-\Omega^{2}\right)\right]\right]
\end{aligned}
$$

The substitution of the real and imaginary parts of these equations into the real part of the dispersion equation shows that only $\mathrm{N}_{3}$ represents a surface wave.

In region III for $0.6 \leq N^{2} \leq 0.9, N_{1}$, as discussed earlier, represents a surface wave but it is now necessary to see if $\mathrm{N}_{2}$ and/or $\mathrm{N}_{3}$ also represent surface waves. The expressions required are

$$
\begin{aligned}
& N_{2 / 3}=\frac{-\left(1-\Omega^{2}\right)}{\left(2 \Omega^{2}-1\right)}-1+2 i \Omega^{2} \xi\left(1-\Omega^{2}\right)^{\frac{3}{2}} /\left(2 \Omega^{2}-1\right)^{\frac{5}{2}}+i \Omega n /\left(2 \Omega^{2}-1\right)^{2} \\
& \alpha_{2 / 3}= \pm\left\{\Omega^{3} n /\left(2 \Omega^{2}-1\right)^{\frac{3}{2}}-1+\Omega^{4} \xi\left(1-\Omega^{2}\right)^{\frac{1}{2}} /\left(2 \Omega^{2}-1\right)^{2}+i\left(1-\Omega^{2}\right) /\left(2 \Omega^{2}-1\right)^{\frac{1}{2}}\right. \\
& \delta_{2 / 3}= \pm\left\{\Omega n\left(1-\Omega^{2}\right) /\left(2 \Omega^{2}-1\right)^{\frac{3}{2}}-1+\Omega^{2} \xi\left(1-\Omega^{2}\right)^{\frac{3}{2}} /\left(2 \Omega^{2}-1\right)^{2}+i \Omega^{2} /\left(2 \Omega^{2}-1\right)^{\frac{1}{2}}\right\} \\
& \gamma_{2 / 3}= \pm\left(1-\Omega^{2}\right)^{\frac{3}{2}} / \xi\left[1-i n\left(2 \Omega^{2}-1\right) / 2 \Omega\left(1-\Omega^{2}\right)\right]
\end{aligned}
$$

Note here that the decay function, in the vacuum is, in fact, $Q\left(K^{2}-\Omega^{2}\right)^{\frac{1}{2}}$ and not $R\left[\left(K^{2}-\Omega^{2}\right)\left(\frac{\Omega+i n}{\Omega}\right]^{2}\right.$ that has been used for convenience upto now. This decay function, for $\mathrm{N}_{2 / 3}$, becomes

$$
Q\left(K_{2 / 3}^{2}-\Omega^{2}\right)^{\frac{1}{2}}=\frac{3}{2} n \Omega /\left(2 \Omega^{2}-1\right)^{3 / 2}-1+\Omega^{2} \xi\left(1-\Omega^{2}\right)^{3 / 2} /\left(2 \Omega^{2}-1\right)^{2}
$$

The use of equation (4.13) leads to the strongest requirement, however, with the automatic fulfillment of (4.15). This requirement is for the real part of equation (4.13) with the positive sign, and the use of $\mathrm{N}_{2}$, to be greater than zero. It is not difficult now to show that the $N_{3}$ root does not represent a surface wave but $N_{2}$ does. Therefore in region III in addition to $N_{1}$ there is a branch corresponding to $N_{2}$. This branch only exists provided that

$$
\eta>\frac{\Omega \xi\left(1-\Omega^{2}\right)^{\frac{1}{2}}}{\left(2 \Omega^{2}-1\right)^{\frac{1}{2}}}
$$


This restriction on $n$ and $\xi$ controls the appearance on the dispersion diagram of the familiar bend-back loop, in competition with spatial dispersion. Typically in the bend-back regionseno.71 $\rightarrow 0.72$ so that the criterion (4.16) reduces to the simple rule $n \geq 6 \xi$. This, assuming that it is qualitatively useful even close to the $\Omega^{2} \simeq 0.5$, gives a useful guide as to whether the bend-back loop can exist or not. (4.16) is of course always satisfied when $\xi=0$ and is never satisfied when $\eta=0$.

$V$ - NUMERICAL ANALYSIS

Region II in the vicinity of $\Omega^{2} \approx 0.5$ does not yield to analytical analys is so it has to be investigated computationally. In Fig. l,2 and 3 are shown computer-generated graphs of the real parts of $\mathrm{N}_{1}, \mathrm{~N}_{2}$ and $\mathrm{N}_{3}$. These correspond to continuations into region II of the previous analytical analysis. A particular feature in Fig.2 is the 'kink' developed in $N_{1}$ as $n$ increases. This shows clearly the interaction between the spatial dispersion branch, which always exists, and the collision-induced bend-back curve. fig.3 shows what happens when the collisions play a more dominant role.

The translation of the analytical and computational analysis into $\left(\Omega, K_{r}\right)$ diagrams is shown in Figs. 4 and 5 for typical values. Here on display is the fact that, when the criterion $n \geqslant 6 \xi$ breaks down, the bend-back loop is isolated, weakened and eventually suppressed leaving only the $N_{1}$ spatial dispersion branch in existence.

\section{VI - CONCLUSION}

We have presented a very detailed analysis of the effect of collisions on the spatially dispersive plasmon-polariton mode. Through a combination of analytical and computational analysis it is shown that although the commonly accepted bend-back can occur it can also be suppressed by the spatial dispersion. It is hoped that this paper will form the basis for a wider discussion of this problem.

\section{VII - REFERENCES}

1. CLEMMOW P.C. and ELGIN J., J.P7asma Phys. 12 (1974) 91.

2. KOVENER G.S., ALEXANDER R.W. and BELL R.J., Phys.Rev.B14 (1976)1458.

3. HALEVI P., "Electromagnetic Surface Modes", John Wiley, Ed. A.D.Boardman (1982) 271 .

4. BOARDMAN A.D., "Electromagnetic Surface Modes", John Wiley, A.D.Boardman (1982) 25. 
5. FERGUSON P., WALLIS R.F., BELAKHOVSKY M. and TOMKINSON J., Surf. Science 76 .

6. OTtO A., Z.Phys. 216 (1968) 398. 